\title{
émulations
}

\section{Ronald van Kempen, Gideon Bolt et Maarten van Ham (dir.) - Neighborhood Decline}

Thomas Kirszbaum

Émulations - Revue de sciences sociales

2020, Comptes rendus critiques, En ligne.

\section{Article disponible à l'adresse suivante}

https://ojs.uclouvain.be/index.php/emulations/article/view/kirszbaum

\section{Pour citer cet article}

Thomas Kirszbaum, « Ronald van Kempen, Gideon Bolt et Maarten van Ham (dir.) Neighborhood Decline », Émulations, en ligne. Mise en ligne le 4 décembre 2020. DOI : 10.14428/emulations.cr.090

Distribution électronique : Université catholique de Louvain (Belgique) : ojs.uclouvain.be

(C) Cet article est mis à disposition selon les termes de la Licence Creative Commons Attribution, Pas d'Utilisation Commerciale 4.0 International. http://creativecommons.org/licenses/by-nc/4.0/

Éditeur : Émulations - Revue de sciences sociales / Presses universitaires de Louvain https://ojs.uclouvain.be/index.php/emulations

ISSN électronique : 1784-5734

PUL PRESSES 


\title{
Ronald van Kempen, Gideon Bolt et Maarten van Ham (dir.) - Neighborhood Decline
}

\author{
Thomas Kirszbaum ${ }^{1}$
}

Recensé: Ronald van Kempen, Gideon Bolt et Maarten van Ham (dir.), Neighborhood Decline, Abingdon/New York, Routledge, 2018, 134 p.

Depuis plus d'un siècle, le quartier est sinon un objet d'étude à part entière, du moins une échelle d'analyse privilégiée par les sciences sociales (Authier, Bacqué, GuérinPace, 2007). Les enquêtes des réformateurs sociaux à la fin du XIX siècle, puis les travaux de l'École de Chicago envisageaient le quartier comme un espace fondamental pour l'intégration des classes laborieuses et des communautés ethniques (Grafmeyer, Joseph, 1979), mais aussi comme un lieu de dysfonctionnements sociaux ${ }^{2}$. Dans les années 1950 et 1960, urbanistes, géographes et sociologues dénonçaient la logique d’aménagement des villes qui, associée à la spéculation foncière, semblait signer la disparition du quartier traditionnel, riche de sa diversité et de l'intensité de ses relations sociales, au profit de grands ensembles ou d'un habitat périurbain fustigés comme des cités-dortoirs mortifères (Coing, 1966 ; Jacobs, 1961 ; Whyte, 1957). À l'ère de la mondialisation contemporaine, la mort du quartier a été une nouvelle fois annoncée : plus mobiles et individualistes, les citadins s'affranchiraient de leurs ancrages territoriaux pour s'inscrire dans des réseaux globalisés, diminuant d'autant l'importance du quartier comme espace de vie structurant les rapports sociaux (Ascher, 1998).

Si l'éloge funèbre du quartier a été maintes fois prononcé, sa capacité de résistance a pourtant été soulignée, à la fois comme lieu de ressources et de production identitaire pour ses habitants, mais aussi comme catégorie de l'action publique, notamment dans les politiques de la ville et de développement communautaire (Authier, Bacqué, GuérinPace, 2007). Le coup de grâce aura-t-il finalement été porté par la crise financière de 2008 et la récession qui l'a suivie, débouchant sur une austérité budgétaire continue, un accroissement de la pauvreté et une fragilisation des systèmes de logements aidés ? Rassemblant les contributions de chercheur-e.s de cinq pays (Pays-Bas, Allemagne, Royaume-Uni, Suède et États-Unis) œuvrant dans les disciplines de la géographie, de

\footnotetext{
${ }^{1}$ Chercheur associé à l'Institut des sciences sociales du politique, ISP, France.

${ }^{2}$ Les études pionnières de l'École de Chicago préfiguraient en cela les travaux plus contemporains (cette fois à dominante économétrique) sur les «effets de quartier » en situation de pauvreté concentrée ; par exemple, ceux de Robert J. Sampson (2012).
} 
l'aménagement et de l'urbanisme, le livre Neighborhood Decline ne mène pas à une conclusion aussi radicale. L'attention prêtée aux effets des contextes nationaux, métropolitains et locaux, ainsi qu'aux interactions complexes entre ces échelles, conduit les auteur·ess à formuler un constat plus nuancé: si la crise tend de façon générale à accentuer la ségrégation des groupes sociaux et ethno-raciaux, elle se combine avec d'autres facteurs, externes et internes aux quartiers, conduisant à différencier la trajectoire de ces derniers.

L'enjeu principal de l'ouvrage est ainsi de comprendre par quels mécanismes la situation des quartiers les moins bien positionnés dans la stratification socio-urbaine peut se dégrader ou au contraire résister aux effets d'une détérioration de leur environnement économique. Si les auteur·e·s usent de bien des précautions méthodologiques, préférant les hypothèses aux constats, ils délivrent néanmoins un message politique : plus durement frappés que tout autre territoire par la crise et la récession qui sévissent depuis une dizaine d'années, ces quartiers devraient faire l'objet d'une attention plus soutenue des gouvernements. Mais ces derniers se retirent de la scène urbaine pour enjoindre les habitants à trouver des solutions locales à des problèmes dont l'origine est, au moins en partie, globale. Si déclin du quartier il y a, comme le suggère le titre du livre, il est assurément repérable dans les politiques publiques des États.

\section{L'impact de la crise économique : hypothèses et points aveugles de la re- cherche}

Les dynamiques de transformation des quartiers (neighborhood change) sont un thème classique de la recherche, notamment dans les pays anglophones ${ }^{3}$. Les travaux scientifiques se sont jusqu'à présent focalisés sur deux figures opposées du changement : la gentrification et la dégradation (decline). Les tentatives de théorisation les plus connues de la gentrification sont celle de Phillip Clay (1979), qui l'a analysée comme un processus par étapes, de l'arrivée des pionniers jusqu'à l'embourgeoisement complet d'un quartier, et celle de Neil Smith (1996) qui, à travers sa théorie du rent gap, voit dans la gentrification une conséquence de la logique capitaliste de rentabilisation de l'espace urbain. Le thème de la dégradation des quartiers a également fait l'objet d'analyses nombreuses, oscillant le plus souvent entre l'exploration de ses causes physiques ${ }^{4}$, socio-économiques (Wilson, 1987) ou raciales (Massey, Denton, 1993).

Aucun de ces deux grands schèmes explicatifs ne donne entièrement satisfaction aux auteur.e.s de Neighborhood Decline. Au lieu d'une approche causale et linéaire, inévitablement réductionniste, les deux contributrices allemandes à l'ouvrage, Katrin Grossmann et Annegret Haase, empruntent à Gilles Deleuze et Félix Guattari (1980) le concept d'agencements collectifs, qui permettrait de rendre compte des interactions mutuelles entre « les gens, les réseaux, les organisations, les bâtiments, mais aussi les

\footnotetext{
${ }^{3}$ Pour une revue de la littérature, voir Ruth Lupton et Anne Power (2004).

${ }^{4}$ Par exemple, l'incrimination de l'architecture moderniste chez Oscar Newman (1972).
} 
politiques publiques, l'environnement législatif et les valeurs » (p. 86). Il s'agit ainsi de prêter attention aux contextes locaux, à la manière dont ils s'imbriquent avec des processus globaux, aux sentiers de dépendance et aux bifurcations soudaines de la trajectoire des quartiers. Ces auteures tentent d'appliquer cette approche à deux quartiers de Leipzig, l'un en voie de gentrification, l'autre en déclin démographique, mais elles reconnaissent que leur analyse localisée, certes détaillée et nuancée, fait perdre de vue les dynamiques structurelles, communes à différents quartiers.

Le chapitre de Derek Hyra et Jacob S. Rugh consiste à comparer la trajectoire de trois quartiers afro-américains en voie de gentrification, localisés dans trois villes différentes (Bronzeville à Chicago, Harlem à New York et Shaw/U Street à Washington, D.C.). S'ils soulignent l'impact de la crise des subprimes, la conclusion principale des auteurs est que ces quartiers ont réagi différemment à la crise : les caractéristiques sociales et raciales des gentrifiers 5 , ainsi que le contexte économique des villes et agglomérations d'appartenance, ont joué selon les cas un rôle d'amplificateur ou d'amortisseur.

Ce chapitre est le seul à proposer des résultats empiriques fondés sur une analyse à la fois longitudinale et comparative, de nature à apprécier les effets différenciés de la crise et de la récession sur les quartiers. Les autres chapitres du livre énoncent plutôt des hypothèses et pistes de recherche pour de futurs travaux empiriques. La contribution des trois coordonnateurs néerlandais de l'ouvrage, Ronald Van Kempen 6 , Gideon Bolt et Maarten van Ham, avance pas moins de dix hypothèses sur l'influence des niveaux macro, méso et micro. Toutes ces hypothèses convergent vers une idée centrale : la crise et la récession accentuent la concentration de ménages pauvres et/ou appartenant à des minorités ethno-raciales dans les mêmes quartiers, risquant d'entraîner ceux-ci dans un processus de dégradation défini comme « toute évolution négative des conditions physiques, démographiques ou socio-économiques d'un quartier, telle qu'éprouvée par ses habitants ou d'autres parties prenantes » (p. 13).

Ce risque serait avéré non seulement aux États-Unis, où les dommages causés par les foreclosures (saisies immobilières) sont déjà bien documentés, mais aussi dans les pays européens héritant d'une forte tradition d'intervention publique, où les régimes d'Étatprovidence apparaissent fragilisés par l'austérité budgétaire et la privatisation croissante du logement aidé. Portant sur les effets de la récession économique du début des années 1990 dans la ville de Malmö en Suède, la contribution de Roger Andersson et Lina Hedman vient confirmer ce point : la récession de 1990 s'était accompagnée d'une

\footnotetext{
${ }^{5}$ Les prêts à risque et les saisies immobilières ont eu une intensité moindre à Harlem et Shaw/U Street dans un contexte de gentrification racialement mixte, en comparaison de Bronzeville où la gentrification est le fait de classes moyennes afro-américaines davantage exposées à ces pratiques bancaires.

${ }^{6}$ Ce géographe néerlandais est décédé en 2016 peu avant la publication de l'ouvrage, dédié à sa mémoire.
} 
polarisation plus forte des revenus, se traduisant par une ségrégation sociale et ethnique accrue de l'espace urbain. Les auteur.e-s restent toutefois prudents, évoquant des corrélations plutôt que des liens de causalité entre ces phénomènes.

À lire les différentes contributions, la compréhension des trajectoires différenciées des quartiers reste encore très lacunaire. La littérature sur le "neighborhood change » identifie des facteurs de changement aux niveaux macro (politiques budgétaires et du crédit immobilier), méso (dynamisme des économies régionales) et micro (qualité du stock de logements, mobilités résidentielles, cohésion sociale des quartiers), mais reste globalement muette concernant les interactions mutuelles entre ces niveaux. De l'aveu de ses auteur.e-s, Neighborhood Decline ne comble que très partiellement cette lacune, mais il a le mérite d'ouvrir le débat.

\section{Mobilité résidentielle et mobilité sociale}

Le livre ouvre une discussion particulièrement heuristique sur les mobilités résidentielles et sociales. Écartant tout déterminisme physique, il nous rappelle que ce sont d'abord (mais pas seulement) les habitants qui façonnent un quartier en décidant de s'y installer, de le quitter ou de s'y maintenir, avec des marges de choix inégales selon leurs ressources socio-économiques. Ce phénomène dit de «mobilités sélectives » est bien connu à propos des quartiers d'habitat social français : les catégories socialement les mieux dotées sont surreprésentées parmi les ménages sortants, tandis qu'ils sont remplacés par de ménages entrants globalement plus pauvres que ceux qui y vivent déjà (Mas, 2017). Dans des marchés du logement segmentés, la mobilité différentielle des groupes sociaux a donc des effets ségrégatifs puissants, soulignés de longue date dans des travaux américains (Grigsby et al., 1987) et désormais français (Lelévrier, 2010 ; Maurin, 2007; Rudolph, 2017).

Un intérêt majeur de Neighborhood Decline est de proposer de croiser cette variable des mobilités résidentielles avec celle la mobilité sociale (descendante en l'occurrence) des ménages actuels du quartier, dont l'importance grandit dans le contexte post-crise de 2008, laquelle, si l'on en croit les auteur·e·s, aurait ralenti la mobilité résidentielle (du moins en Europe). Il se pourrait donc que la dégradation d'un quartier reflète d'abord l'accroissement du chômage et de la pauvreté dans la société en général.

Voilà qui interroge les politiques de redistribution sociale et qui interpelle aussi les politiques de renouvellement urbain. Celles-ci ont cherché jusqu'à présent à revaloriser les quartiers populaires par la mobilité résidentielle (soit le relogement plus ou moins forcé des habitants en place et l'attraction de nouveaux ménages) davantage que par la promotion socio-économique des habitants en place. S'il ne s'agit que de dessiner le cahier des charges de futures recherches, on ne peut qu'approuver l'appel des auteur.e.s à mesurer l'influence respective des mobilités résidentielles et de la mobilité sociale sur les dynamiques d'évolution des quartiers et d'ajuster les politiques publiques en conséquence. 


\section{Le quartier, catégorie déclinante des politiques publiques}

L'accentuation de la ségrégation urbaine sous l'effet de la récession économique aurait dû logiquement conforter l'assise des politiques de la ville et de développement communautaire. C'est pourtant le schéma inverse qui s'observe dans les différents pays : les politiques ciblées sur des quartiers désavantagés refluent partout, certes à cause de la raréfaction des ressources publiques, mais aussi parce qu'elles sont frappées de discrédit. C'est le cas de la politique de la ville française, même si ce pays n'est pas traité dans l'ouvrage (Epstein, Kirszbaum, 2019). C'est le cas également au Royaume-Uni, comme le montre la contribution de Rebecca Tunstall. L'auteure interprète le désenchantement vis-à-vis de la politique urbaine comme le fruit du décalage entre les attentes élevées à son endroit et la forte inertie des quartiers. L'évaluation intensive de la National Strategy for Neighborhood Renewal, la politique la plus ambitieuse menée au cours des deux dernières décennies au Royaume-Uni, a révélé que les quartiers ayant fait l'objet de la plus forte attention par les pouvoirs publics avaient certes connu des améliorations, mais que leur position relative dans la hiérarchie sociale et urbaine n'avait guère évolué. Le pas a été vite franchi, du côté des gouvernants, pour conclure à l'inefficacité de ce type de programmes urbains.

Le discrédit de la politique urbaine prend une forme différente aux États-Unis, mais aboutit pareillement à dévaloriser le quartier comme échelle pertinente de l'action publique. Tel est l'objet du chapitre rédigé par Kathe Newman et Edward Goetz. OutreAtlantique, le quartier reste la pierre angulaire des pratiques dites de développement communautaire (community development $t^{7}$ ), mais celles-ci subissent tout à la fois les effets de la globalisation économique et de la concurrence de l'approche métropolitaine (regionalism). La crise des subprimes a révélé combien la circulation du capital à l'ère de la globalisation financière pouvait dramatiquement refaçonner l'histoire d'un quartier, condamnant ses acteurs à l'impuissance. L'efficacité des démarches communautaires pour inverser les dynamiques de déclin dans les quartiers urbains est également mise en doute par les tenants de l'approche dite régionaliste qui font de l'agglomération la seule échelle véritablement pertinente du traitement de la ségrégation urbaine. Tout en pointant les limites d'une action par trop focalisée sur l'échelle des quartiers, Newman et Goetz critiquent les présupposés de cette dernière approche - la pathologisation des quartiers pauvres - et ses conséquences pratiques, à savoir la démolition et le déplacement des habitants vers d'autres quartiers. On l'a vu, cette stratégie, également mise en œuvre en Europe (quoique de façon moins radicale), est largement inefficace quand il s'agit d'améliorer la condition socio-économique des habitants restés sur place.

Mobilisant le concept de «politique du lieu » (politics of place), définie comme la politique s'efforçant d'infléchir les processus multiscalaires qui façonnent les espaces

${ }^{7}$ La « community » est à entendre ici au sens du quartier et des acteurs qui évoluent à cette échelle. 
(Pierce, Martin, Murphy, 2011), les deux auteur.e-s dessinent ce que pourrait être l'agenda du développement communautaire au XXI $\mathrm{XI}^{\mathrm{e}}$ siècle. Il s'agit de renouer avec l'esprit radical et de confrontation qui animait ses acteurs dans les années 1960, dont les auteur·e·s estiment, à la suite de bien d'autres, qu'il s'est émoussé depuis lors dans une approche à la fois consensuelle et néolibérale. Newman et Goetz invitent les praticiens du développement communautaire à se mobiliser pour peser sur les processus globaux (y compris la régulation financière) qui conditionnent le bien-être des quartiers, tout en continuant d'agir à cette échelle afin de répondre aux besoins les plus immédiats de leurs habitants. Sont citées en ce sens la Coalition pour le droit à la ville (Right to the City Coalition) ou les « analyses de l'équité régionale " (regional equity analysis) qui consistent à dresser la carte des ressources accessibles par les habitants des quartiers pauvres et à interpeller les décideurs sur cette base.

Ce livre ouvre ainsi de nombreuses pistes tant pour la recherche que pour l'action. Sa limite principale est inhérente à nombre d'ouvrages collectifs qui, en rassemblant des contributions hétérogènes, rendent difficile la lecture d'une thèse centrale. Ce défaut est sans doute accentué dans le cas de Neighborhood Decline qui reprend l'intégralité d'un dossier de la revue anglophone Urban Geography paru sous le même titre et avec le même contenu en 2016. Il faut donc apprécier ce livre pour ce qu'il est : un numéro de revue où le lecteur puisera analyses et informations au gré de ses intérêts, à défaut d'y trouver une thèse solidement charpentée, que l'on s'est efforcé de reconstituer en partie ici.

\section{Bibliographie}

AsChER F. (1998), La République contre la ville. Essai sur l'avenir de la France urbaine, La Tour d'Aigues, Éditions de l'Aube (« Monde en cours »).

Authier J.-Y., BACQue M.-H., Guerin-PACE F. (2007), Le quartier. Enjeux scientifiques, actions politiques et pratiques sociales, Paris, La Découverte ("Recherches »).

CLAY P. L. (1979), Neighborhood Renewal, Lexington, Lexington Books.

CoING H. (1966), Rénovation urbaine et changement social, Paris, Les Éditions ouvrières («Évolution de la vie sociale »).

Deleuze G., Guattari F. (1980), Capitalisme et schizophrénie 2. Mille plateaux, Paris, Éditions de Minuit («Critique»).

EPSTEIN R., KiRSZBAum T. (2019), "Ces quartiers dont on préfère ne plus parler. Les métamorphoses de la politique de la ville (1977-2018) », Parlement[s], Revue d'histoire politique, vol. 30, $n^{\circ} 3$, p. 23-46. 
Grafmeyer Y., Joseph I. (1979), L'École de Chicago. Naissance de l'écologie urbaine, Paris, Champ urbain.

Grigsby W., Baratz M., Galster G., Maclennan D. (1987), "The Dynamic of Neighborhood Change and Decline », Progress in Planning, vol. 28, $\mathrm{n}^{\circ}$ 1, p. 1-76.

JACOBS J. (1961), The Death and Life of Great American Cities, New York, Vintage Books.

LELEVRIER C. (2010), "La mixité dans la rénovation urbaine: dispersion ou reconcentration?», Espaces et sociétés, vol. 140-141, n 1-2, p. 59-74.

LuPton R., Power A. (2004), What We Know About Neighbourhood Change: A Literature Review, CASE Report $\mathrm{n}^{\circ} 27$.

MAS S. (2017), «Mobilité résidentielle en quartiers prioritaires de la politique de la ville », in ONPV, Rapport annuel de l'Observatoire national de la politique de la ville, Paris, CGET.

Massey D. S., Denton N. A. (1993), The American Apartheid. Segregation and the Making of the Underclass, Chicago, University of Chicago Press.

MAURIN É. (2007), «La ségrégation urbaine, son intensité et ses causes », in S. Paugam (dir.), Repenser la solidarité. L'apport des sciences sociales, Paris, Presses universitaires de France (« Le lien social »), p. 621-633.

Newman O. (1972), Defensible Space. Crime Prevention through Urban Design, New York, Macmillan.

Pierce J., Martin D. G., Murphy J. T. (2011), « Relational Place-Making: The Networked Politics of Place», Transactions of the Institute of British Geographers, vol. 36, n 1 , p. 54-70.

Rudolph M. (2017), «Ceux qui partent, ceux qui restent. Les mobilités résidentielles dans les villes en décroissance», Métropolitiques. En ligne. URL: www.metropolitiques.eu/Ceux-qui-partent-ceux-qui-restent.html.

SAMPSON R. J. (2012), Great American City. Chicago and the Enduring Neighborhood Effect, Chicago, University of Chicago Press.

Smith N. (1996), The New Urban Frontier: Gentrification and the Revanchist City, New York/Londres, Routledge.

Whyte W. H. (dir.) (1957), The Exploding Metropolis, New York, Doubleday.

Wilson W. J. (1987), The Truly Disadvantaged. The Inner City, the Underclass, and Public Policy, Chicago, University of Chicago Press. 
THOMAS KIRSZBAUM

ÉMULATIONS, LECTURE CRITIQUE, EN LIGNE, 2020 